\title{
Aerobic Oxidative Cleavage of Vicinal-Diol Fatty Esters by Supported Ruthenium Hydroxide Catalyst
}

Boris Guicheret, ${ }^{a}$ Eric Da Silva, ${ }^{a}$ Regis Philippe, ${ }^{b}$ Alain Favre-Reguillon,,$b$ Laurent Vanoye, ${ }^{b}$ Philippe Blach, ${ }^{d}$ Yann Raoul, ${ }^{d}$ Claude De Bellefon, ${ }^{b}$ Estelle Métay ${ }^{* a}$ and Marc Lemaire*a

a Univ Lyon, Université Claude Bernard Lyon1, CNRS, INSA-Lyon, CPE-Lyon, Institut de Chimie et Biochimie Moléculaires et Supramoléculaires, ICBMS, UMR 5246, Equipe CAtalyse, SYnthèse et ENvironnement (CASYEN), Campus LyonTech La Doua, Bâtiment Lederer, 1 rue Victor Grignard, 69100 Villeurbanne

b Univ Lyon, CNRS, Université Claude-Bernard Lyon 1, CPE-Lyon, Laboratoire de Génie des Procédés Catalytiques (LGPC-UMR 5285), F-69616, VILLEURBANNE, France

c Conservatoire National des Arts et Métiers, EPN 7, 292 rue Saint Martin, 75003 Paris

d OLEON, AVRIL group, Rue les Rives de l'Oise BP 20609 - Compiègne

Corresponding author: estelle.metay@univ-lyon1.fr

Contents:

Number of pages: 11

Number of Figures: 13

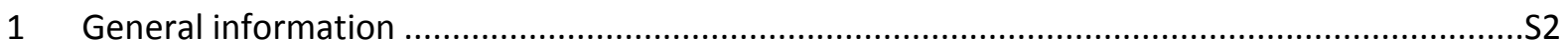

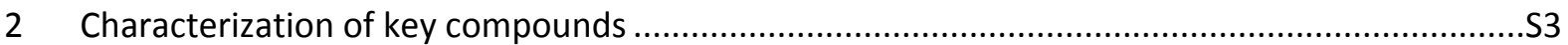

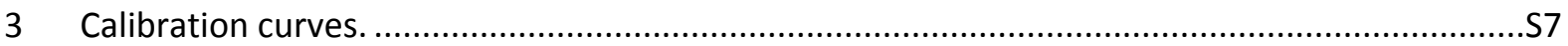

4 Oxidative cleavage of $6 \mathrm{~b}$ by $\mathrm{Ru}(\mathrm{OH})_{x} / \gamma-\mathrm{Al}_{2} \mathrm{O}_{3}$ catalyst $\left(6\right.$ mol\%) using $\mathrm{O}_{2}$ as oxidant.................S10

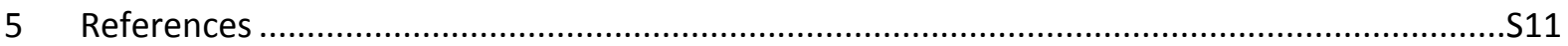




\section{General information}

All reagents were used as received from the suppliers: Sigma Aldrich, Alfa Aesar, and TCl. All reagents and reactants were used without further purification.

${ }^{1} \mathrm{H}$ NMR and ${ }^{13} \mathrm{C}$ NMR spectra were recorded on a BRUKER DRX 300 or BRUKER ALS $300\left({ }^{1} \mathrm{H} 300\right.$ $\mathrm{MHz},{ }^{13} \mathrm{C} 75 \mathrm{MHz}$ ) in $\mathrm{CDCl}_{3}$ (except when mentioned) and chemical shifts are given in ppm. $\mathrm{CDCl}_{3}{ }^{1} \mathrm{H}-$ NMR: 7.26ppm; ${ }^{13} \mathrm{C}-\mathrm{NMR}: 77.16 \mathrm{ppm}$, DMSO ${ }^{1} \mathrm{H}-\mathrm{NMR}: 2.50 \mathrm{ppm} ;{ }^{13} \mathrm{C}-\mathrm{NMR}: 39.5 \mathrm{ppm}, J$ values are given in $\operatorname{Hertz}(\mathrm{Hz})$. Abbreviations are defined as follows: $\mathrm{s}=$ singlet, $\mathrm{d}=$ doublet, $\mathrm{t}=$ triplet, $\mathrm{m}=$ multiplet, $\mathrm{br}=$ broad. IR spectra were recorded on a Spectro Nicolet IS10 Smart ITR with an ATR diamond and frequencies are given in $\mathrm{cm}^{-1}$.

The HRMS-ESI mass spectra were recorded in a positive ion mode on a hybrid quadrupole time-offlight mass spectrometer (MicroTOFQ-II, Bruker Daltonics, Bremen, Germany) with an Electrospray Ionization (ESI) ion source. The gas flow of spray gas is 0.6 bar and the capillary voltage is $4.5 \mathrm{kV}$. The solutions are infused at $180 \mu \mathrm{L} / \mathrm{h}$ in a mixture of solvents (methanol/dichloromethane/water $45 / 40 / 15)$. The mass range of the analysis is $50-1000 \mathrm{~m} / \mathrm{z}$ and the calibration was done with sodium formate. The mass HRMS-Cl spectra were recorded on a double-focusing mass spectrometer (Thermo Finnigan MAT95XL, Bremen, Germany) equipped with a chemical ionization (Cl) source.

The GC-MS analysis was performed on Focus GC (Thermo Electron Corporation, Bremen, Germany) equipped with a DB-5MS capillary column $(30 \mathrm{~m}, 0.25 \mathrm{~mm}$ i.d, $0.25 \mu \mathrm{m}$ film thickness) and a DSQ mass spectrometer as a detector (Thermo Electron Corporation, Bremen, Germany). The carrier gas was helium, at a flow rate of $1 \mathrm{~mL} / \mathrm{min}$. The column temperature was initially $70^{\circ} \mathrm{C}$ for $2 \mathrm{~min}$, then gradually increased to $310^{\circ} \mathrm{C}$ at $15^{\circ} \mathrm{C} / \mathrm{min}$ and finally at $310^{\circ} \mathrm{C}$ for $10 \mathrm{~min}$. The injector temperature was $220^{\circ} \mathrm{C}$ and the transfer line temperature was $280^{\circ} \mathrm{C}$. For GC-MS detection an electron ionization 


\section{Characterization of key compounds}

Compounds $\mathbf{6 a}$ and $\mathbf{6 b}$ were prepared and purified according to $\mathrm{M}$. Lemaire et $a .^{1}{ }^{1}$ Compounds $\mathbf{8}$, the mixture of $\mathbf{1 1 a}$ and $\mathbf{1 1 b},{ }^{2}$ and compound $\mathbf{1 2}$ were prepared and purified according to M. Lemaire et al. ${ }^{2}$ Compounds $\mathbf{2 a}, \mathbf{3 a}, \mathbf{7}, \mathbf{1 3}$, and $\mathbf{1 4}$ are commercially available from Sigma Aldrich and were used without further purification.

Compounds 9 and $\mathbf{1 0}$ were obtained from $\mathbf{6 b}$ under neat conditions using air as oxidant and $\mathrm{Ru}(\mathrm{OH}) \mathrm{x} / \gamma-\mathrm{Al}_{2} \mathrm{O}_{3}$. Purification of the crude on silica gel using EtOAc/heptane as eluant.

Compound 9 : Methyl 8-(2, 5-dioctyl-1, 3-dioxolan-4-yl)octanoate, $\mathrm{C}_{28} \mathrm{H}_{54} \mathrm{O}_{4}, 454.40 \mathrm{~g} / \mathrm{mol}$<smiles>[R]OC(=O)C1OC2OC1C(C(C)C)C2[Y]</smiles>

Colorless oil, IR (ATR) $\sigma_{\max }=2923,2853,1741,1117 \mathrm{~cm}^{-1} .{ }^{1} \mathrm{H}-\mathrm{NMR}\left(300 \mathrm{MHz} ; \mathrm{CDCl}_{3}\right), \delta=0.84(\mathrm{t}, \mathrm{J}=6.9$ $\left.\mathrm{Hz} 6 \mathrm{H}, 2 \mathrm{CH}_{3}\right), 1.24-1.58\left(\mathrm{~m}, 40 \mathrm{H}, \mathrm{CH}_{2}\right), 2.29\left(\mathrm{t}, J=7.5 \mathrm{~Hz}, 2 \mathrm{H}, \mathrm{CH}_{2}\right), 3.51\left(\mathrm{br}, 2 \mathrm{H}, \mathrm{CH}-\mathrm{O}_{\text {ketal }}\right) 3.63(\mathrm{~s}, 3 \mathrm{H}$, $\left.\mathrm{CH}_{3}\right), 4.91\left(\mathrm{~m}, 1 \mathrm{H}\left(\mathrm{CH}_{\text {ketal }}\right) \cdot{ }^{13} \mathrm{C}-\mathrm{NMR}\left(\mathrm{CDCl}_{3} ; 75 \mathrm{MHz}\right) \delta=14.1\left(\mathrm{CH}_{3}\right), 22.7\left(2 \mathrm{CH}_{2}\right), 24.1\left(\mathrm{CH}_{2}\right), 25.0\left(\mathrm{CH}_{2}\right)\right.$, $26.1\left(2 \mathrm{CH}_{2}\right), 29.1-29.7\left(10 \mathrm{CH}_{2}\right), 31.9\left(2 \mathrm{CH}_{2}\right), 33.2\left(2 \mathrm{CH}_{2}\right), 34.1\left(\mathrm{CH}_{2}\right), 34.5\left(\mathrm{CH}_{2}\right) 51.4\left(\mathrm{CH}_{3}\right.$ ester $), 80.1$, $82.2\left(\mathrm{CH}-\mathrm{O}_{\text {ketal }}\right) 103.2\left(\mathrm{CH}-\mathrm{O}_{\text {ketal }}\right) 174.5\left(\mathrm{C}_{\text {ester }}\right)$.

HRMS-ESI: $m / z[M H]^{+}$calculated for: $\left[\mathrm{C}_{28} \mathrm{H}_{54} \mathrm{NaO}_{4}\right]: 477.3920$, found: 477.3906 .

Compound 10 : Dimethyl 8-8'-(5-octyl-1, 3-dioxolan-2, 4-diyl)dioctanoate, $\mathrm{C}_{29} \mathrm{H}_{54} \mathrm{O}_{6}, 498.39 \mathrm{~g} / \mathrm{mol}$

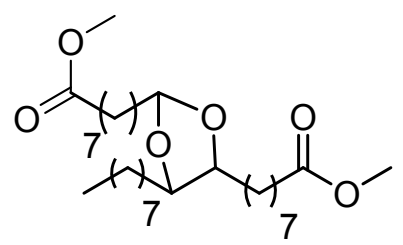

Colorless oil, IR (ATR) $\sigma_{\max }=2925,2854,1738,1458,1168,724 \mathrm{~cm}^{-1} \cdot{ }^{1} \mathrm{H}-\mathrm{NMR}\left(300 \mathrm{MHz} ; \mathrm{CDCl}_{3}\right), \delta=$ $0.80\left(\mathrm{t}, J=6.9 \mathrm{~Hz} 3 \mathrm{H}, \mathrm{CH}_{3}\right), 1.19-1.53\left(\mathrm{~m}, 38 \mathrm{H}, \mathrm{CH}_{2}\right), 2.21\left(\mathrm{t}, \mathrm{J}=7.5 \mathrm{~Hz}, 4 \mathrm{H}, \mathrm{CH}_{2}\right), 3.47\left(\mathrm{br}, 2 \mathrm{H}, \mathrm{CH}-\mathrm{O}_{\text {ketal }}\right)$ $3.58\left(\mathrm{~s}, 6 \mathrm{H}, \mathrm{CH}_{3}\right), 4.86\left(\mathrm{~m}, 1 \mathrm{H}\left(\mathrm{CH}_{\text {ketal }}\right) .{ }^{13} \mathrm{C}-\mathrm{NMR}\left(\mathrm{CDCl}_{3} ; 75 \mathrm{MHz}\right) \delta=14.1\left(\mathrm{CH}_{3}\right), 22.6,23.9,24.9,26.0\right.$, 29.0-29.5 $\left(9 \mathrm{CH}_{2}\right), 31.8\left(\mathrm{CH}_{2}\right), 33.1\left(2 \mathrm{CH}_{2}\right), 34.0\left(2 \mathrm{CH}_{2}\right), 34.4 .\left(\mathrm{CH}_{2}\right) 51.3\left(2 \mathrm{CH}_{3}\right.$ ester $), 80.9,82.1(\mathrm{CH}-$ $\left.\mathrm{O}_{\text {ketal }}\right) 103.0$ (CH-ketal) 174.1 (2 $\left.\mathrm{C}_{\text {ester }}\right)$.

HRMS-ESI: $m / z[\mathrm{MH}]^{+}$calculated for: $\left[\mathrm{C}_{29} \mathrm{H}_{54} \mathrm{NaO}_{6}\right]: 521.3818$, found: 521.3802 . 


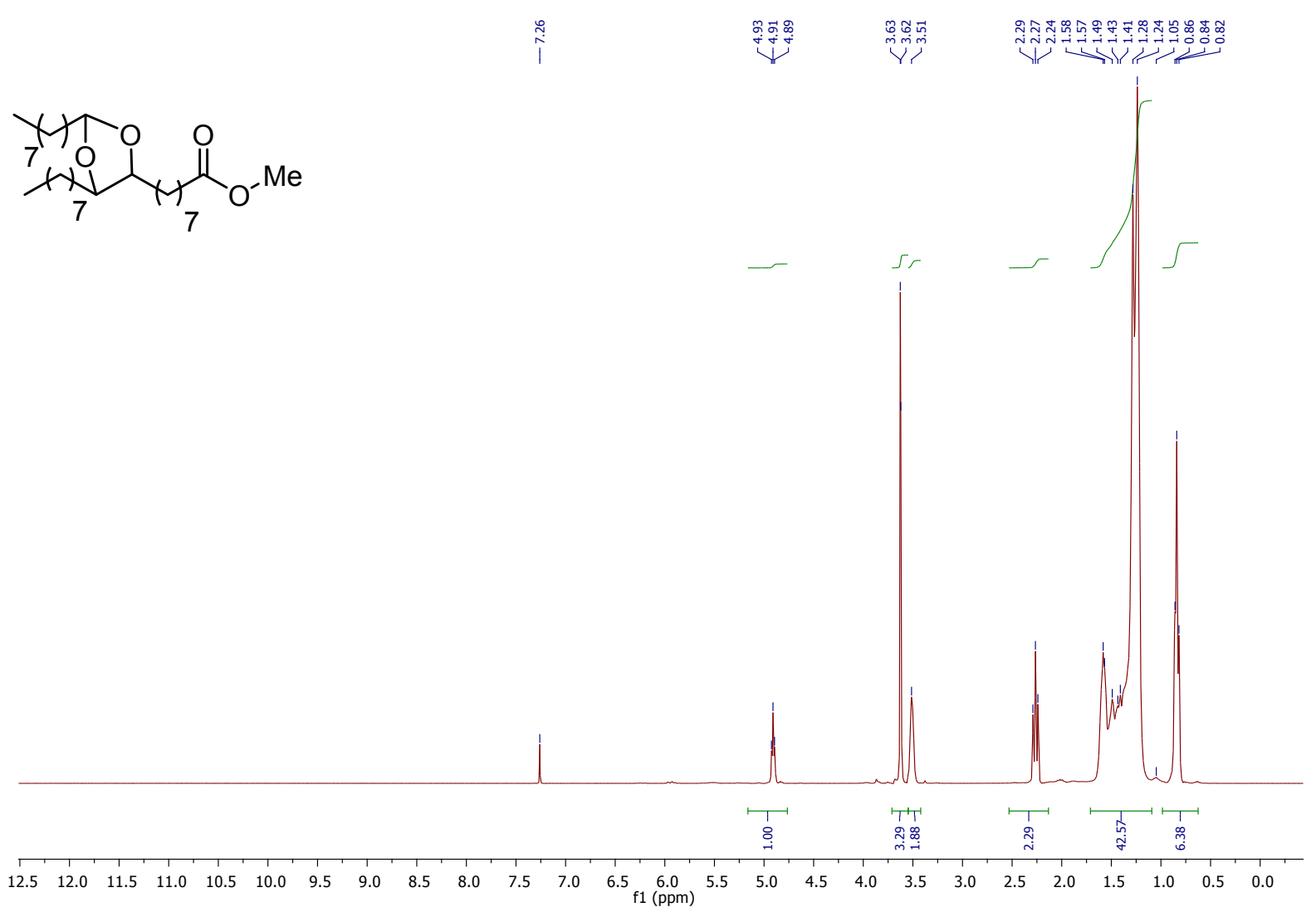

Figure S 1: ${ }^{1} \mathrm{H} N \mathrm{NR}$ of compound 9
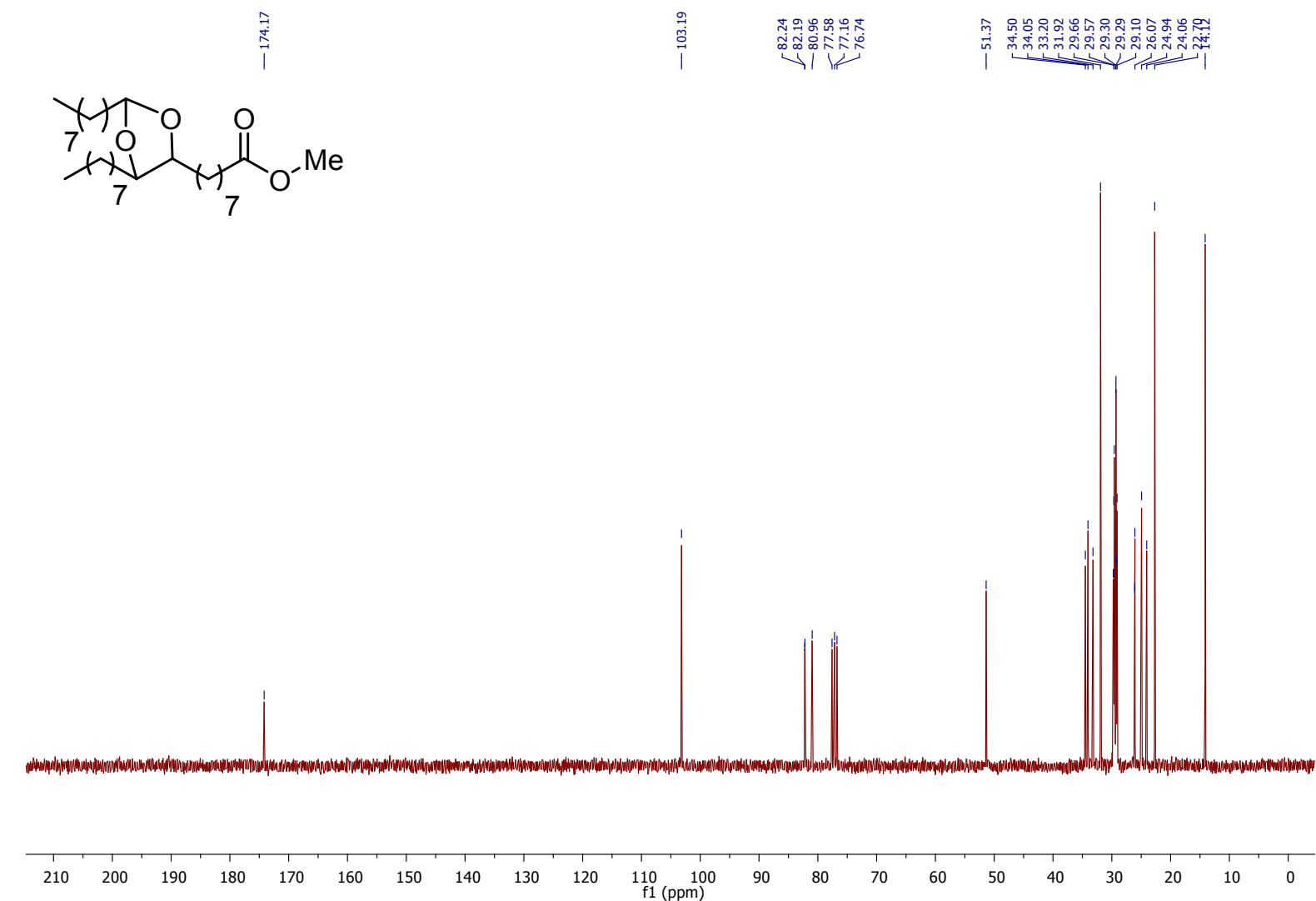

Figure S 2: ${ }^{13} \mathrm{C} N M R$ of compounds 9 


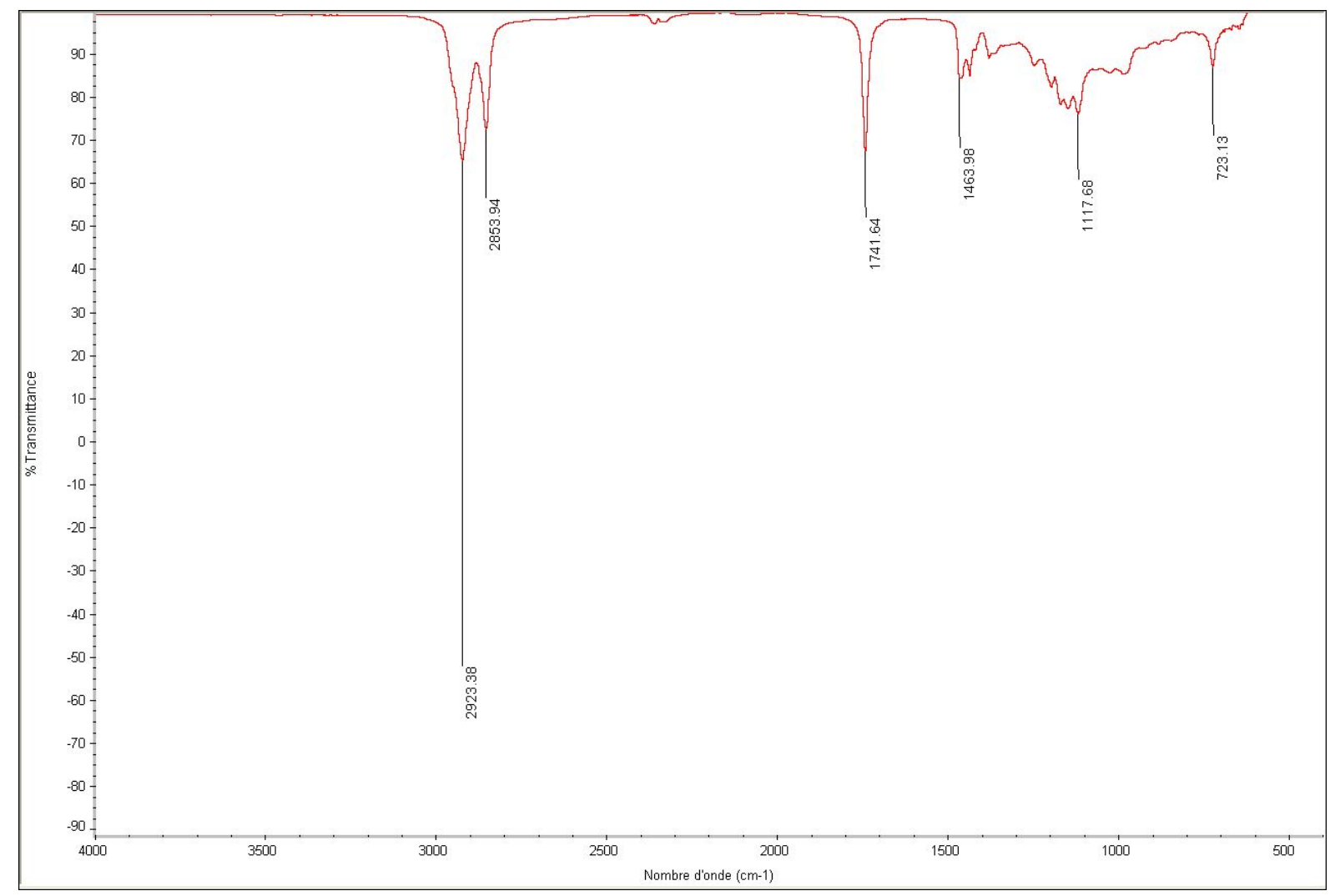

Figure S 3: IR of compound 9

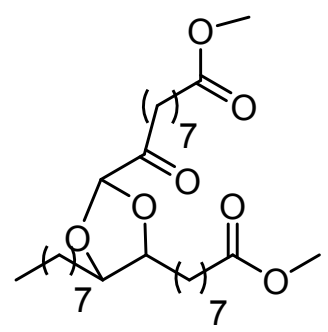

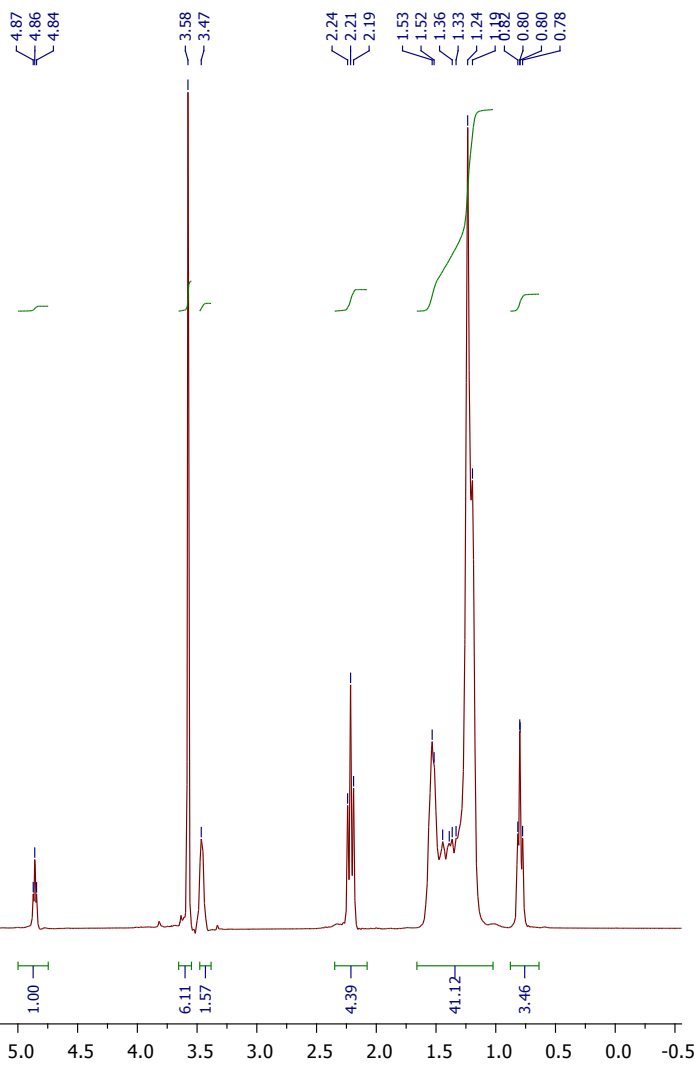

Figure S 4: ${ }^{1} \mathrm{H}$ NMR of compound 10 

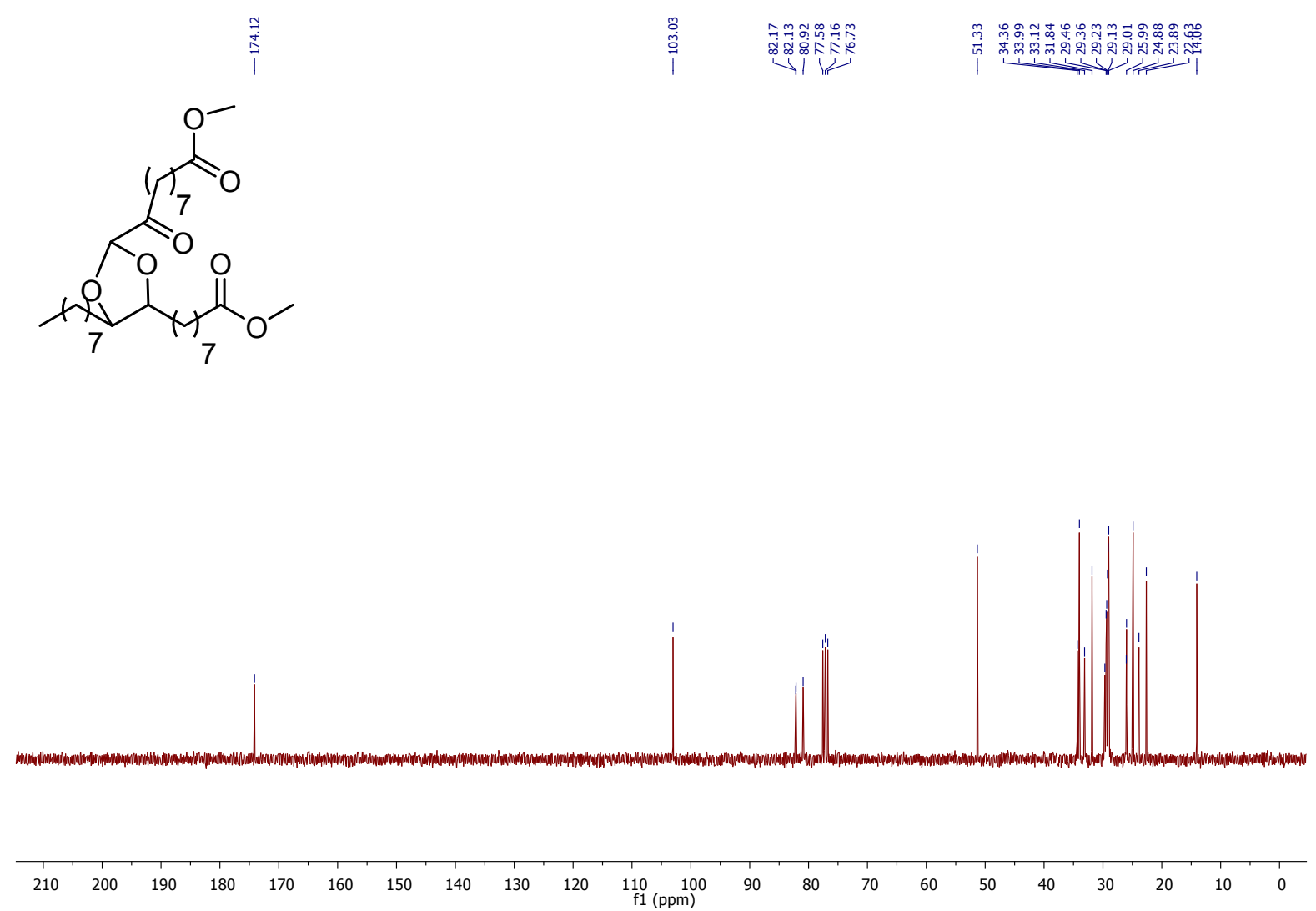

Figure S 5: ${ }^{13} \mathrm{C}$ NMR of compound 10

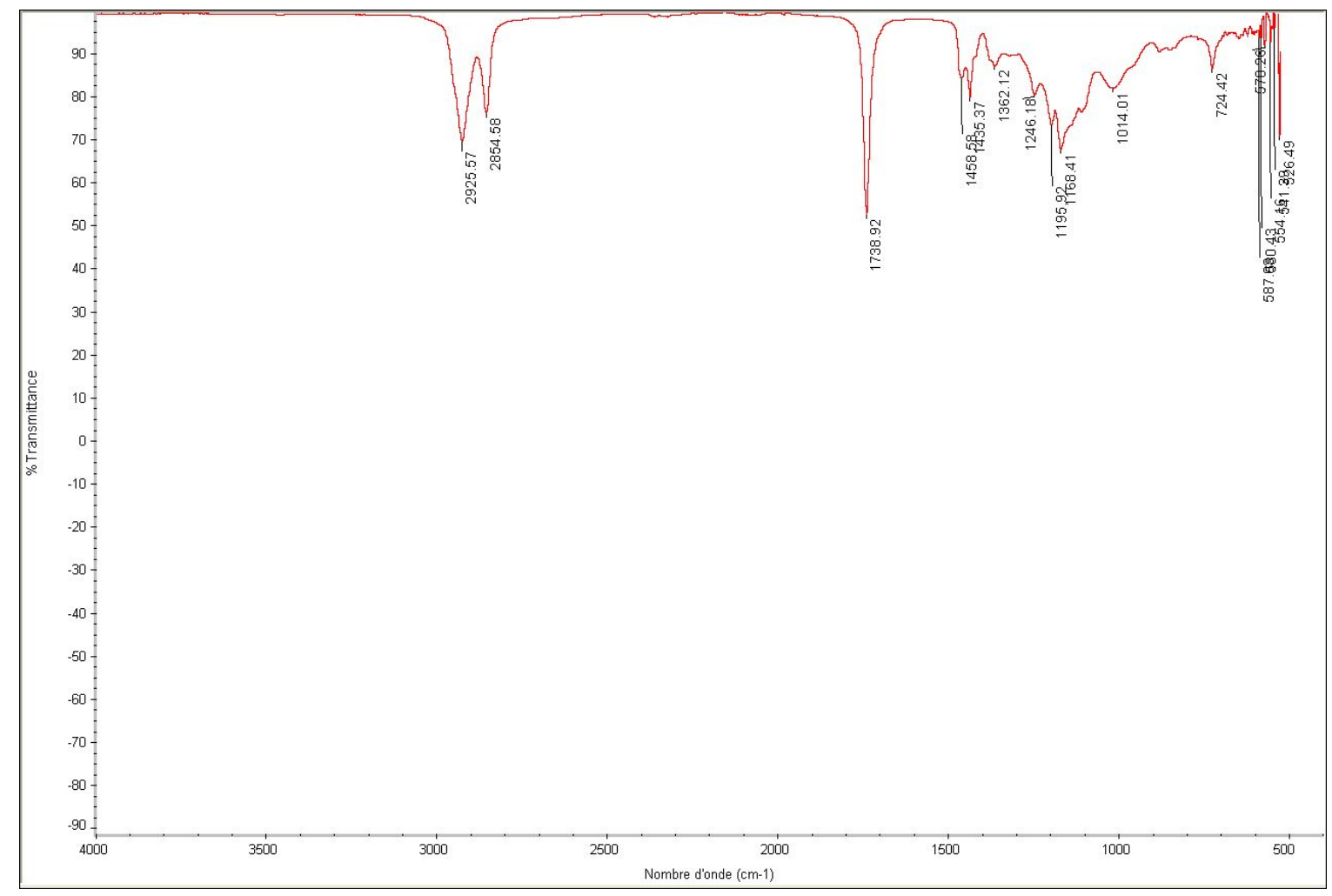

Figure S 6: IR of compound 10 


\section{Calibration curves.}

Calibration curves were obtained in EtOAc using eicosan as an external standard [Eicosan] $=804 \mathrm{mg} / \mathrm{L}$.

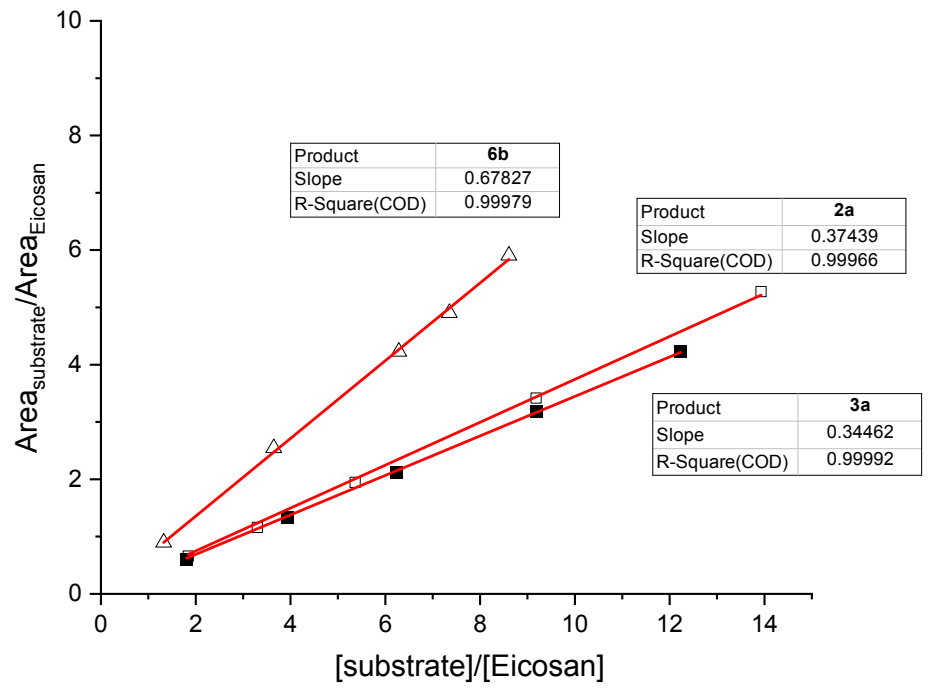

Figure S 7. Calibration curve of compounds $6 \mathbf{b}, \mathbf{2} a$, and $\mathbf{3 a}$

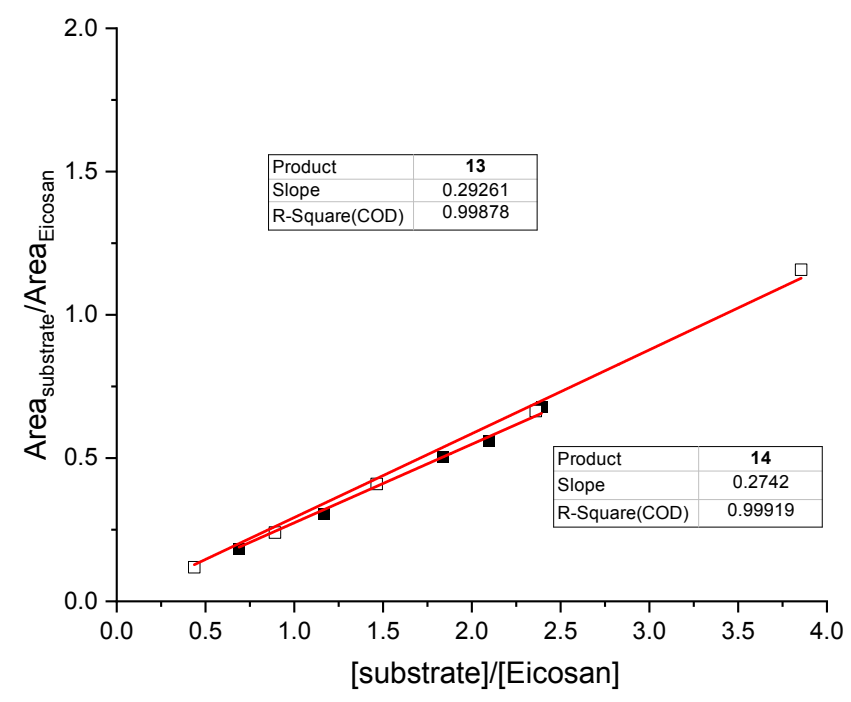

Figure S 8. Calibration curve of compounds 13 and 14 


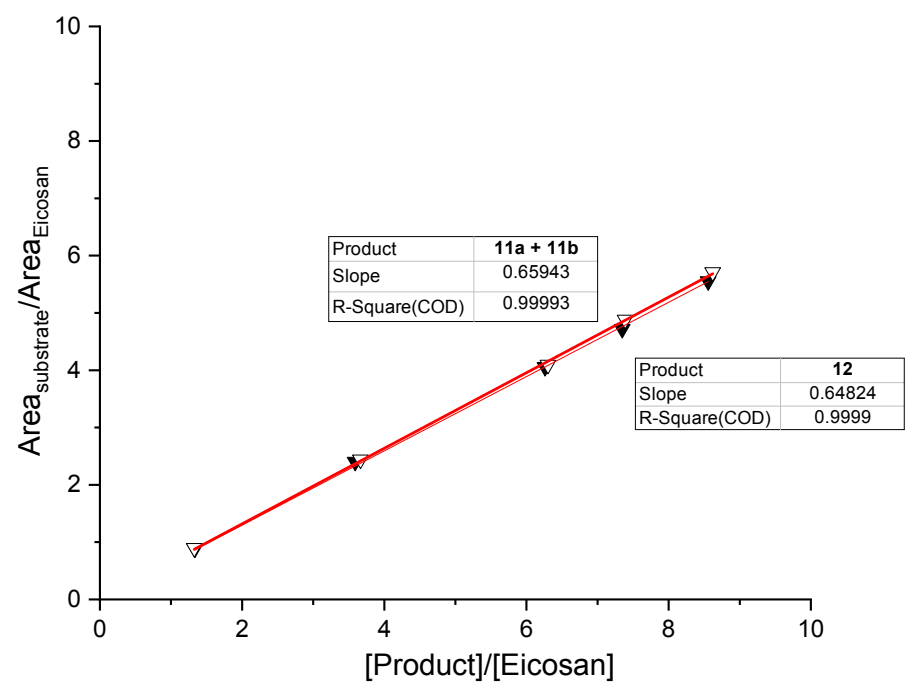

Figure S 9. Calibration curve of compounds $11(11 a+11 b)$ and 12

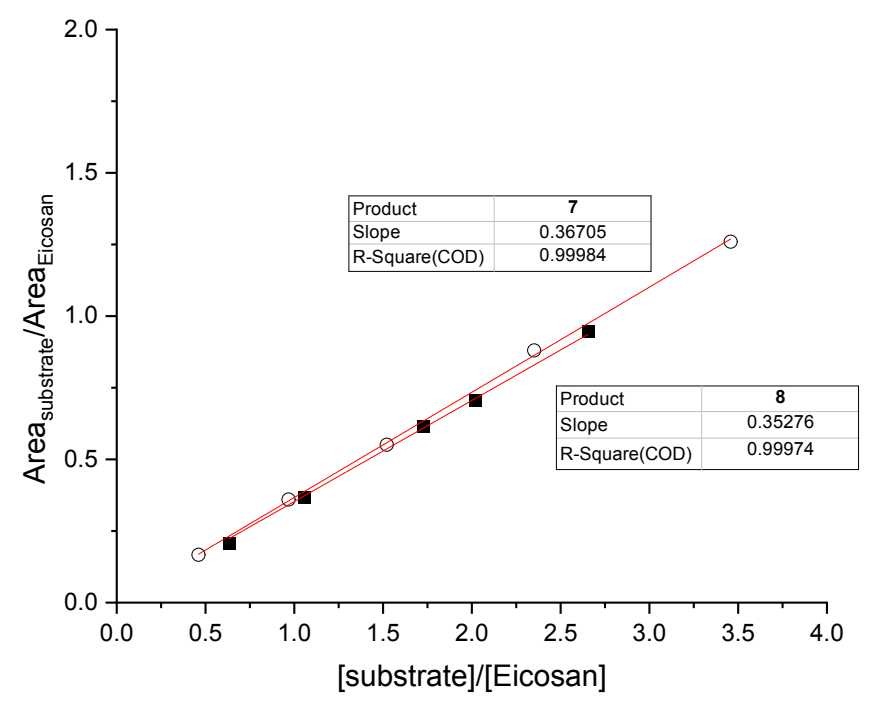

Figure S 10. Calibration curve of compounds 7 and 8 


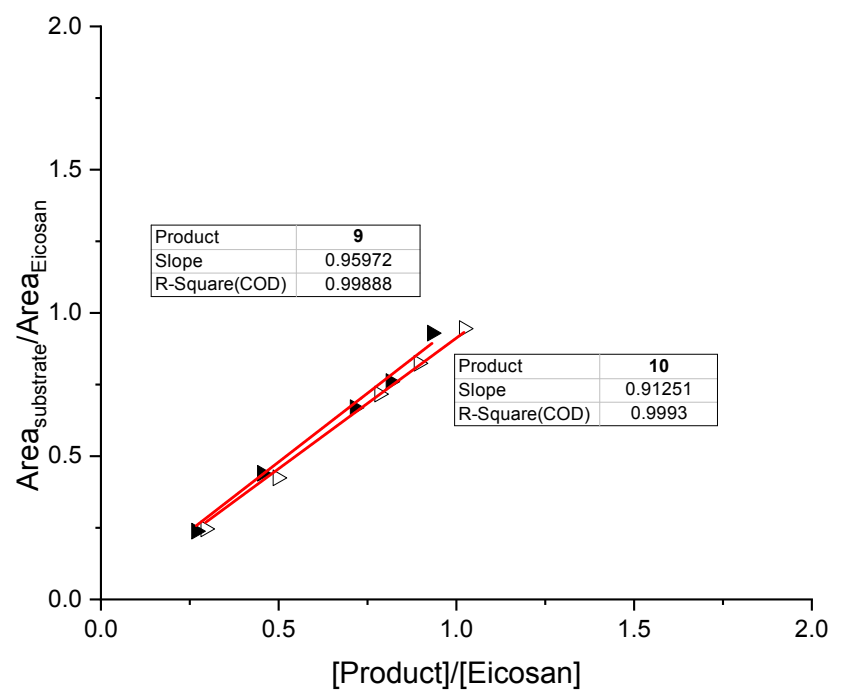

Figure S 11. Calibration curve of compounds 9 and 10 
4 Oxidative cleavage of $6 \mathbf{b}$ by $\mathrm{Ru}(\mathrm{OH})_{x} / \gamma-\mathrm{Al}_{2} \mathrm{O}_{3}$ catalyst (6 mol\%) using $\mathrm{O}_{2}$ as oxidant.

Concentration of $[6 \mathrm{~b}]=33 \mathrm{~g} / \mathrm{l}$ in $\mathrm{H}_{2} \mathrm{O}, 150^{\circ} \mathrm{C}, \mathrm{O}_{2} 5$ bar.

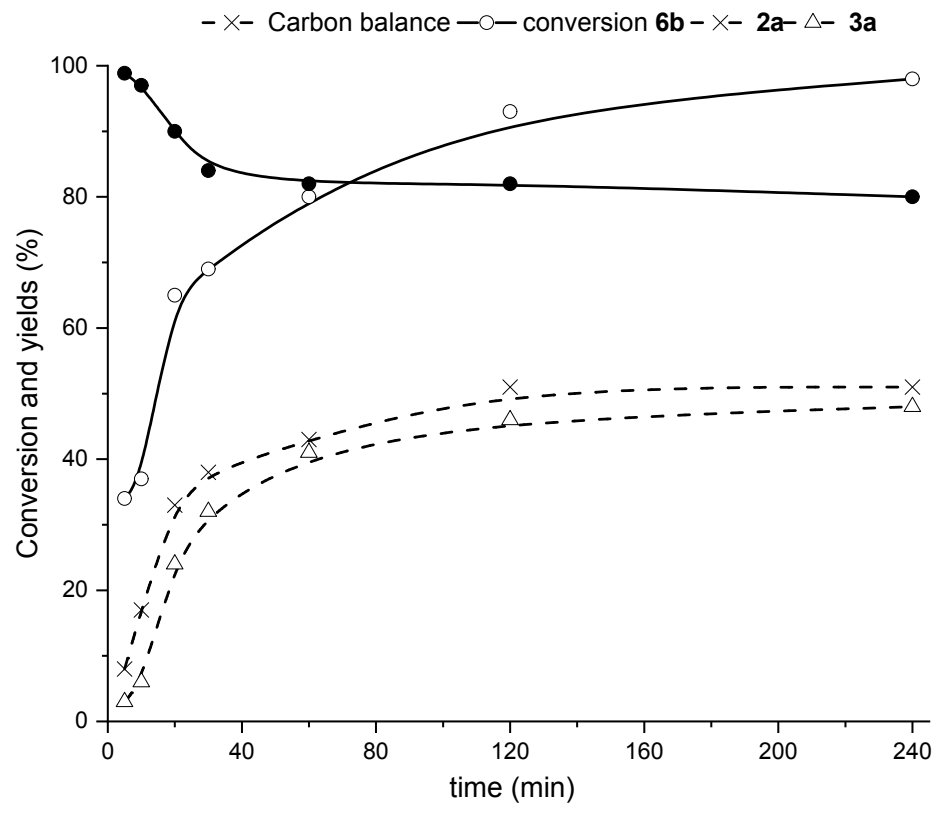

Figure S 12. Evolution of the conversion of $\mathbf{6 b}$, carbon balance, and yield of the products $\mathbf{2} \mathbf{a}$ and $\mathbf{3 a}$ as a function of time during the oxidative cleavage of $6 \mathbf{b}$ by $\mathrm{Ru}(\mathrm{OH})_{\times} / \gamma-\mathrm{Al}_{2} \mathrm{O}_{3}$ catalyst ( 6 mol\%) using $\mathrm{O}_{2}$ as oxidant. [6b] $=33 \mathrm{~g} / \mathrm{L}$ in $\mathrm{H}_{2} \mathrm{O}, 150^{\circ} \mathrm{C}, \mathrm{O}_{2} 5$ bar. Yields are determined by GC after calibration with eicosan as an external standard. 


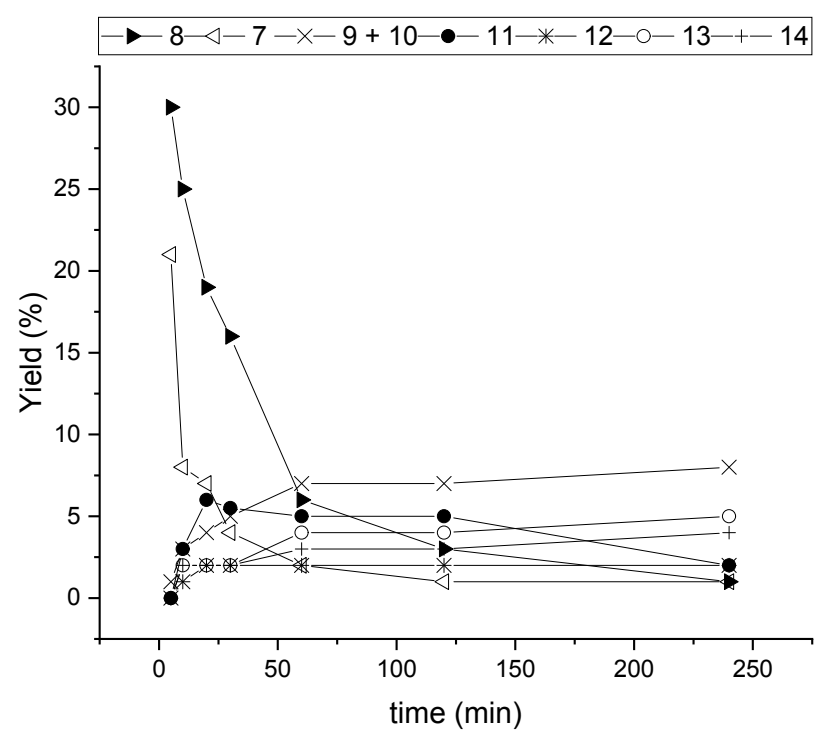

Figure $S$ 13. Evolution of the yield of the products $8,7,9+10,11,12,13$, and 14 as a function of time during the oxidative cleavage of $6 \mathbf{b}$ by $\mathrm{Ru}(\mathrm{OH})_{\times} / \gamma-\mathrm{Al}_{2} \mathrm{O}_{3}$ catalyst $(6 \mathrm{~mol} \%)$ using $\mathrm{O}_{2}$ as oxidant. [6b]=33g/L in $\mathrm{H}_{2} \mathrm{O}, 150^{\circ} \mathrm{C}, \mathrm{O}_{2} 5$ bar. Yields are determined by $\mathrm{GC}$ after calibration with eicosan as an external standard.

\section{References}

1. Guicheret, B.; Bertholo, Y.; Blach, P.; Raoul, Y.; Metay, E.; Lemaire, M., A two-step oxidative cleavage of 1,2-diol fatty esters into acids or nitriles by a dehydrogenation-oxidative cleavage sequence ChemSusChem 2018, 11 (19), 3431-3437, DOI 10.1002/cssc.201801640.

2. Vu, N. D.; Bah, S.; Deruer, E.; Duguet, N.; Lemaire, M., Robust organocatalysts for the cleavage of vegetable oil derivatives to aldehydes through retrobenzoin condensation Chem. Eur.J. 2018, 24 (32), 8141-8150, DOI 10.1002/chem.201800091. 